\title{
A NOTE ON THE FOURIER TRANSFORM OF FRACTAL MEASURES
}

\author{
M. BURAK ERdỡan
}

\section{Introduction}

Let $\mu$ be a compactly supported non-negative measure in $\mathbf{R}^{d}$. For $\alpha \in(0, d)$, the $\alpha$-dimensional energy of $\mu$ is defined via (see, e.g., [2])

$$
I_{\alpha}(\mu):=\iint \frac{\mathrm{d} \mu(x) \mathrm{d} \mu(y)}{|x-y|^{\alpha}}=c_{\alpha, d} \int \frac{|\widehat{\mu}(\xi)|^{2}}{|\xi|^{d-\alpha}} \mathrm{d} \xi,
$$

where $\widehat{\mu}$ is the Fourier transform of the measure $\mu$ :

$$
\widehat{\mu}(\xi)=\int e^{-i x \cdot \xi} \mathrm{d} \mu(x) .
$$

We are interested in the behavior of the Fourier transform of measures with finite energy. It is easy to see that $I_{\alpha}(\mu)<\infty$ does not imply any pointwise decay of $|\widehat{\mu}(\xi)|$ as $|\xi| \rightarrow \infty$. However, in general, averages of $\widehat{\mu}(\xi)$ behave much better.

Let $\Gamma$ be a smooth submanifold of $\mathbf{R}^{d}$ and let $\nu_{\Gamma}$ be a smooth surface measure on $\Gamma$. One may ask the following general question: Fix $\alpha \in(0, d)$ and assume that $I_{\alpha}(\mu)=1$. For which $\beta>0$

$$
\int_{\Gamma}|\widehat{\mu}(R \xi)|^{2} \mathrm{~d} \nu_{\Gamma}(\xi) \leq C_{\beta} R^{-\beta}
$$

for all $R>1$ ?

The following theorem is a slight generalization of a result in [6]. We include a proof in the appendix for the sake of completeness.

Theorem 1. Let $\mu$ be a non-negative measure supported in the unit ball in $\mathbf{R}^{d}$ with $I_{\alpha}(\mu)=1$. Fix $a, b \in(0, d)$ and let $\nu$ be a compactly supported probability measure which satisfies

$$
|\widehat{\nu}(\xi)| \lesssim|\xi|^{-a} \text { and } \quad \nu(B(x, r)) \lesssim r^{b}, \quad \forall x, \xi \in \mathbf{R}^{d}, \forall r>0 .
$$

Then

$$
\int|\widehat{\mu}(R \xi)|^{2} d \nu(\xi) \lesssim R^{-\max (\min (\alpha, a), \alpha-d+b)}
$$

Received August 15, 2003. 
The case $\Gamma=S^{d-1} \subset \mathbf{R}^{d}$ was investigated by several authors [3], [4], [5], [1], [6] and [10] in connection with the continuum version of the Erdos' distance set problem. In this case, Theorem 1 can be applied with $a=(d-1) / 2$ and $b=d-1$ but does not give sharp bounds for all $\alpha$. Sharp bounds for each $\alpha$ are known only in dimension 2, see [10]. We discuss the result of [10] in more detail below. In higher dimensions, the known results are slightly better than the bounds given by Theorem 1, see [1].

The general case that $\Gamma$ has non-vanishing Gaussian curvature was investigated in [6].

In [10], Wolff obtained the following bound: Fix $\alpha \in[1,2]$, and assume that $I_{\alpha}(\mu)=1$. Then for each $\varepsilon>0$

$$
\int_{S^{1}}|\widehat{\mu}(R \xi)|^{2} \mathrm{~d} \nu(\xi) \leq C_{\varepsilon} R^{-\alpha / 2+\varepsilon}, \quad \forall R>1 .
$$

This bound is sharp modulo $R^{\varepsilon}$, c.f. [5]. Sharp bounds for $\alpha \in(0,1]$ are given by Theorem 1 and were first obtained by Mattila [4].

As pointed out in [4], (2) implies that any compact set $E \subset \mathbf{R}^{2}$ with Hausdorff dimension $>4 / 3$ has a positive measure distance set, $\Delta(E)=\{|x-y|: x, y \in E\}$.

By the uncertainty principle and duality, (2) follows from the following theorem (see Lemma 1.5 in [10] and the discussion following it). Let $A_{R}(1)$ be the annulus $\left\{x \in \mathbf{R}^{2}: R-1<|x|<R+1\right\}$.

Theorem 2. ([10]) Let $\alpha \in[1,2]$. Let $\mu$ be a probability measure supported in the unit ball in $\mathbf{R}^{2}$. Assume that

$$
\mu(B(x, r)) \leq C_{1} r^{\alpha} \text { for all } x \in \mathbf{R}^{2} \text { and } r>1 / R .
$$

Let $f$ be a function supported in $A_{R}(1)$ with $L^{2}$ norm 1 . Let $G=f^{\vee}$ be its inverse Fourier transform. Then for all $\varepsilon>0$ and $R>1$

$$
\left|\int G d \mu\right| \leq C_{\varepsilon} C_{1}^{1 / 2} R^{\frac{1}{2}-\frac{\alpha}{4}+\varepsilon} .
$$

In the first part of the paper, we give a different proof of Wolff's result and extend it in the following direction. The following theorem can be considered as a weighted version of the Stein-Tomas restriction theorem.

Theorem 3. Let $\alpha \in[1,2]$. Let $\mu$ be a non-negative measure supported in the unit ball in $\mathbf{R}^{2}$ which satisfies (3). Let $f$ be a function supported in $A_{R}(1)$ with $L^{2}$ norm 1. Let $G=f^{\vee}$. Then, for all $q \geq 1$ and $R>1$, we have

$$
\|G\|_{L^{q}(\mu)} \leq C_{s, q} C_{1}^{1 / q} R^{s}, \quad \forall s>\max \left(\frac{1}{2}-\frac{\alpha}{4}, \frac{1}{4}+\frac{1-\alpha}{2 q}, \frac{1}{2}-\frac{\alpha}{q}\right) .
$$

Moreover, if $\mu\left(\mathbf{R}^{2}\right) \leq 1$, then for all $q \in[1,2]$ and $R>1$, we have

$$
\|G\|_{L^{q}(\mu)} \leq C_{\varepsilon} C_{1}^{1 / 2} R^{\frac{1}{2}-\frac{\alpha}{4}+\varepsilon}, \forall \varepsilon>0 .
$$


Remark 1. The range of $s$ in (5) is sharp modulo endpoint issues. To prove the necessity of the condition $s \geq \frac{1}{4}+\frac{1-\alpha}{2 q}$, let $f_{1}$ be an $L^{2}$ normalized smooth bump function supported in the rectangle $\left\{x \in \mathbf{R}^{2}:\left|x_{2}\right|<R^{1 / 2},\left|x_{1}-R\right|<\right.$ $1 / 2\} \subset A_{R}(1)$ which satisfies i) $\left|f_{1}^{\vee}(x)\right|>R^{1 / 4} / 100$ for $x \in P=\left\{x \in \mathbf{R}^{2}\right.$ : $\left.\left|x_{1}\right|<1,\left|x_{2}\right|<R^{-1 / 2}\right\}$, ii) $f_{1}^{\vee}$ has a Schwartz decay away from $P$. Let $\mathrm{d} \mu(x)=$ $R^{1-\alpha / 2} \chi_{P}(x) \mathrm{d} x$. Note that $\mu$ satisfies (3) with $C_{1} \approx 1$. To prove the necessity of the condition $s \geq \frac{1}{2}-\frac{\alpha}{2}$, let $f_{2}=R^{-1 / 2} \chi_{A_{R}(1)}$, and choose a measure $\mu$ with $\mu\left(B\left(0, R^{-1}\right)\right) \geq R^{-\alpha}$. To prove the condition $s \geq \frac{1}{2}-\frac{\alpha}{4}$, we modify the first example above. Fix $T \approx R^{(\alpha-1) / 2}$ and let

$$
F^{\vee}(x)=T^{-1 / 2} \sum_{k=1}^{T} f_{1}^{\vee}\left(x-\frac{k}{T} e_{2}\right) .
$$

Note that $F$ is supported in $A_{R}(1),\|F\|_{2} \approx 1$, and $\left|F^{\vee}\right| \gtrsim R^{1 / 4} T^{-1 / 2}$ on the set $S=\cup_{k=1}^{T}\left(P+\frac{k}{T} e_{2}\right)$ (because of the Schwartz decay of $\left.f_{1}^{\vee}\right)$. Finally, let $\mathrm{d} \mu(x)=R^{1-\alpha / 2} \chi_{S}(x) \mathrm{d} x$. Note that $\mu$ satisfies (3) with $C_{1} \approx 1$.

The range of $s$ in (6) and the dependence on $C_{1}$ is also sharp modulo endpoints. To see this take the function $f_{1}$ above and let $\mathrm{d} \mu(x)=R^{1 / 2} \chi_{P}(x) \mathrm{d} x$. Note that $\mu$ is a probability measure which satisfies (3) with $C_{1} \approx R^{(\alpha-1) / 2}$.

Remark 2. Note that in the first part of the theorem we don't need any additional assumption on the total mass of $\mu$. The claim (5) for $q \in[1,2)$ follows from the case $q=2$, Hölder's inequality and the bound $\mu\left(\mathbf{R}^{2}\right)=\mu(B(0,1)) \leq C_{1}$ which follows from (3). The second claim follows from the first one in the same way by using the additional assumption $\mu\left(\mathbf{R}^{2}\right) \leq 1$ instead of $\mu\left(\mathbf{R}^{2}\right) \leq C_{1}$. A similar remark is valid for Theorem 5 below.

Remark 3. One can obtain some partial results in higher dimensions analogous to Theorem 3 and Wolff's result (4) by combining the proof of Theorem 3 with the recent parabolic bilinear restriction estimate of Tao [7]. In particular, one can obtain the following partial result in the distance set problem:

$$
E \subset \mathbf{R}^{d} \text {, compact and } \operatorname{dim}(E)>\frac{d(d+2)}{2(d+1)} \Longrightarrow|\Delta(E)|>0 .
$$

The conjectured exponent is $d / 2$, see [3]. Tao's result comes into play in the inequalities (22)-(24) below. Note that (24) is the well-known $L^{2} \times L^{2} \rightarrow L^{2}$ bilinear restriction estimate. One can use Hölder's inequality with $p>(d+2) / d$ and $p^{\prime}$ in (22) instead of Cauchy-Schwarz and then use the $L^{2} \times L^{2} \rightarrow L^{p}$ bilinear restriction estimate of Tao after a parabolic rescaling to estimate the first integral. In fact, one needs a statement which is more general than the main result in [7], namely one needs a bilinear restriction estimate for elliptic surfaces with implicit constants depending on the surface locally uniformly. It is possible to obtain this statement by going through the proof of Theorem 1.1, see the final remark in [7]. We omit the details. 
In the second part of the paper, we consider the problem (1) in the case when $\Gamma$ is a cone in $\mathbf{R}^{3}$. Let $\Gamma=\left\{(x, t) \in \mathbf{R}^{2} \times \mathbf{R}:|x|=t, t \in[1,2]\right\}$. Let $\nu$ be the normalized surface measure on $\Gamma$. Let $\beta(\alpha)$ be the supremum of all $\beta \geq 0$ such that the inequality

$$
\int_{\Gamma}|\widehat{\mu}(R u)|^{2} \mathrm{~d} \nu(u) \leq C_{\sigma} R^{-\beta}
$$

holds for every non-negative measure $\mu$ supported in the unit ball in $\mathbf{R}^{3}$ with $I_{\alpha}(\mu)=1$.

In the appendix, we discuss counterexamples which imply that

$$
\beta(\alpha) \leq \begin{cases}\alpha & , \alpha \in(0,1 / 2] \\ 1 / 2 & , \alpha \in[1 / 2,1] \\ \alpha / 2 & , \alpha \in[1,2] \\ \alpha-1 & , \alpha \in[2,3)\end{cases}
$$

As one may expect, these exponents are same as the exponents for $S^{1}$ for $\alpha<2$.

Note that the bound

$$
\beta(\alpha) \geq \max (\min (\alpha, 1 / 2), \alpha-1)
$$

follows from Theorem 1 . The following theorem takes care of the remaining case $\alpha \in(1,2)$.

Theorem 4. $\beta(\alpha) \geq \frac{\alpha}{2}$ for $\alpha \in(1,2)$.

Theorem 4 follows from the following theorem as in the case of circles.

Let $\Gamma_{R}(C)$ be the $C$-neighborhood of $R \Gamma=\left\{(x, t) \in \mathbf{R}^{2} \times \mathbf{R}:|x|=t, t \in\right.$ $[R, 2 R]\}$. Let

$$
s_{0}(\alpha, q):= \begin{cases}\max \left(1-\frac{\alpha}{4}, \frac{3}{4}-\frac{\alpha-1}{2 q}, 1-\frac{\alpha}{q}\right), & \text { for } \alpha \in[1,2], \\ \max \left(\frac{3-\alpha}{2}, \frac{3}{4}+\frac{3-2 \alpha}{2 q}, 1-\frac{\alpha}{q}\right), & \text { for } \alpha \in(2,3] .\end{cases}
$$

Theorem 5. Let $\alpha \in[1,3]$. Let $\mu$ be a non-negative measure supported in the unit ball. Assume that

$$
\mu(B(x, r)) \leq C_{1} r^{\alpha} \text { for all } x \in \mathbf{R}^{3} \text { and } r>1 / R .
$$

Let $f$ be a function supported in $\Gamma_{R}(1)$ with $L^{2}$ norm 1 . Let $G$ be its inverse Fourier transform. Then for all $q \geq 1$ and $R>1$, we have

$$
\|G\|_{L^{q}(\mu)} \leq C_{s, q} C_{1}^{1 / q} R^{s}, \quad \forall s>s_{0}(\alpha, q) .
$$

Moreover, if $\mu\left(\mathbf{R}^{3}\right) \leq 1$, then for all $q \in[1,2]$ and $R>1$, we have

$$
\|G\|_{L^{q}(\mu)} \leq C_{s} C_{1}^{1 / 2} R^{s}, \quad \forall s>s_{0}(\alpha, 2) .
$$

Using (11), one can obtain the following Strichartz type estimates relative to fractal measures for the wave equation in $2+1$ dimensions. 
Corollary 1. Let $\alpha \in[1,3]$, and let $\mu$ be a non-negative measure supported in the unit ball satisfying $\mu(B(x, r)) \leq r^{\alpha}$ for all $r>0$ and $x \in \mathbf{R}^{3}$. Let $u$ be a solution of

in $\mathbf{R}^{3}$. Then

$$
\square u=0, \quad u(\cdot, 0)=f, \quad \frac{d u}{d t}(\cdot, 0)=g
$$

$$
\|u\|_{L^{q}(d \mu)} \lesssim\|f\|_{H^{s}}+\|g\|_{H^{s-1}}
$$

for all $s>s_{0}(\alpha, q)$. Here $\|f\|_{H^{s}}=\left\|(1-\Delta)^{s / 2} f\right\|_{2}$.

Remark 4. The inequality (12) is already known for $s>\max (3 / 4,1-\alpha / 4,1-$ $\alpha / q$ ) (see [11] (p.1283-1287) for a nice discussion about this type of inequalities).

Remark 5. The range of $s$ in Theorem 5 is sharp modulo endpoints. The counterexamples are similar to the ones given in Remark 1.

Remark 6. For $\alpha \in[2,3]$, the proof of Theorem 5 is relatively easy. Parseval's theorem implies (11) for $q=2$ and $s>\frac{3}{2}-\frac{\alpha}{2}$. On the other hand $L^{2}$ Fourier restriction theory implies (11) for $q=6$ and $s>1-\frac{\alpha}{6}$. It is also easy to see that (11) holds for any $q$ if $s=1$. Interpolating these bounds, we obtain (11).

\section{List of notation}

$\chi_{A}$ : Characteristic function of the set $A$.

$B(x, r):=\{y:|x-y|<r\}$.

$A_{R}(C):=\left\{x \in \mathbf{R}^{2}: R-C<|x|<R+C\right\}$.

$\Gamma_{R}(C):=\left\{(x, t) \in \mathbf{R}^{2} \times \mathbf{R}: t \in[R, 2 R],|| x|-t| \leq C\right\}$.

If $P$ is a rectangle of dimensions $a_{1} \times a_{2} \times \ldots \times a_{d}$ in $\mathbf{R}^{d}$, then

$C P$ is the rectangle of dimensions $C a_{1} \times C a_{2} \ldots \times C a_{d}$ with the same center and axis directions as $P$.

A dual rectangle of $P$ is a rectangle with the same axis directions and dimensions $a_{1}^{-1} \times a_{2}^{-1} \times \ldots \times a_{d}^{-1}$.

$a_{P}$ is a fixed affine map from $\mathbf{R}^{d}$ to $\mathbf{R}^{d}$ which takes the unit cube $Q$ to $P$. $\varphi$ : A fixed Schwartz function (for each dimension $d$ ) which is equal to 1 in $Q$ and vanishes outside $2 Q$. Moreover $\varphi^{\vee}$ satisfies

$$
\left|\varphi^{\vee}(\xi)\right| \leq C_{M} \sum_{j=1}^{\infty} 2^{-M j} \chi_{2^{j} Q}(\xi), \quad \forall \xi \in \mathbf{R}^{d}, \forall M \in \mathbf{Z}^{+} .
$$

$\phi$ : A fixed non-negative Schwartz function satisfying: i) $\phi>1 / 2$ on $Q$, ii) $\widehat{\phi}$ is supported in $Q$, iii) the inequality (13).

$\varphi_{P}:=\varphi \circ a_{P}^{-1}$.

$\phi_{P}:=\phi \circ a_{P}^{-1}$.

$C$ : A constant which may vary from line to line. 
$A \lesssim B: A \leq C B$

$A \approx B: A \lesssim B$ and $B \lesssim A$

$|A|$ : length of the vector $A$ or the measure of the set $A$.

\section{Proof of Theorem 3}

In the proof of the theorem, we make repeated use of the following lemma.

Lemma 3.1. Let $\alpha \in[1,2]$. Let $\mu$ be a non-negative measure in $\mathbf{R}^{2}$ which satisfies (3) with $C_{1}=1$. Let $D$ be a rectangle of dimensions $R_{1} \times R_{2}$ such that $R_{1} \leq R_{2} \lesssim R$. Let $D_{\text {dual }}$ be the dual of $D$ centered at the origin. Then the function $\mu_{D}:=\left|\varphi_{D}^{\vee}\right| * \mu$ satisfies

I) $\left\|\mu_{D}\right\|_{\infty} \lesssim R_{2}^{2-\alpha}$,

II) $\left\|\mu_{D}\right\|_{1} \lesssim 1$

III) $\mu_{D}\left(x+K D_{\text {dual }}\right):=\int_{K D_{\text {dual }}} \mu_{D}(x+y) d y \lesssim K^{\alpha} R_{2}^{1-\alpha} R_{1}^{-1}, \forall K \gtrsim 1$ and $x \in \mathbf{R}^{2}$.

Proof. Fix $M>100$. Using (13), we obtain

$$
\left|\varphi_{D}^{\vee}(x)\right| \lesssim R_{1} R_{2} \sum_{j=1}^{\infty} 2^{-M j} \chi_{2^{j} D_{d u a l}}(x) .
$$

I) Using (14), we obtain

$$
\begin{aligned}
\left|\varphi_{D}^{\vee}\right| * \mu(x) & \lesssim R_{1} R_{2} \sum_{j} 2^{-M j} \int \chi_{2^{j} D_{\text {dual }}}(x-y) \mathrm{d} \mu(y) \\
& \lesssim R_{1} R_{2} \sum_{j} 2^{-M j}\left(2^{j} R_{2}^{-1}\right)^{\alpha} \frac{R_{2}}{R_{1}} \lesssim R_{2}^{2-\alpha} .
\end{aligned}
$$

The second inequality follows from (3) and the observation that $2^{j} D_{\text {dual }}$ can be covered by $\lesssim R_{2} / R_{1}$ many balls of radius $2^{j} R_{2}^{-1}$.

II) follows from Young's inequality using $\left\|\varphi_{D}^{\vee}\right\|_{1} \lesssim 1$.

III) Without loss of generality assume $x=0$. Using (14) and (3) like above, we obtain

$$
\begin{aligned}
\mu_{D}\left(K D_{\text {dual }}\right) & \lesssim R_{1} R_{2} \sum_{j} 2^{-M j} \iint \chi_{K D_{\text {dual }}}(y) \chi_{2^{j} D_{\text {dual }}}(y-u) \mathrm{d} \mu(u) \mathrm{d} y \\
& \lesssim R_{1} R_{2} \sum_{j} 2^{-M j} \iint \chi_{\left(K+2^{j}\right) D_{\text {dual }}}(u) \chi_{2^{j} D_{\text {dual }}}(y-u) \mathrm{d} y \mathrm{~d} \mu(u) \\
& \lesssim R_{1} R_{2} \sum_{j} 2^{-M j}\left[\left(K+2^{j}\right)^{\alpha} R_{2}^{-\alpha} \frac{R_{2}}{R_{1}}\right]\left[\frac{2^{2 j}}{R_{1} R_{2}}\right] \\
& \lesssim K^{\alpha} R_{2}^{1-\alpha} R_{1}^{-1}
\end{aligned}
$$


We also need the following well-known geometric lemma about the size of intersection of circular annuli. Given interval $J \subset[-1 / 2,1 / 2]$, let $A_{R}(C, J)=$ $\left\{(\rho \cos (\theta), \rho \sin (\theta)) \in A_{R}(C): \theta \in J\right\}$.

Lemma 3.2. Let $J_{1}, J_{2} \subset[-1 / 2,1 / 2]$ be two intervals of length $\ell \gtrsim R^{-1 / 2}$. Assume that the distance between $J_{1}$ and $J_{2}$ is $\gtrsim \ell$, then for any $x \in \mathbf{R}^{2}$

$$
\left|\left(x+A_{R}\left(1, J_{1}\right)\right) \cap A_{R}\left(1, J_{2}\right)\right| \lesssim \ell^{-1} .
$$

Proof. In the case $\ell \approx R^{-1 / 2}$ the statement is void since $\left|A_{R}(1, J)\right| \lesssim \ell R \lesssim$ $R^{1 / 2} \lesssim \ell^{-1}$. Assume $\ell>1000 R^{-1 / 2}$, and fix $x, J_{1}, J_{2}$. Note that the set $A_{1} \cap$ $A_{2}:=\left(x+A_{R}\left(1, J_{1}\right)\right) \cap A_{R}\left(1, J_{2}\right)$ has at most two connected components. Let $\mathcal{C}$ be a connected component. It suffices to prove that $\operatorname{diam}(\mathcal{C}) \lesssim \ell^{-1}$. Take a point $y \in \mathcal{C}$. Take an infinite strip $\mathcal{S}_{1}\left(\mathcal{S}_{2}\right.$, resp. $)$ of thickness 10 tangent to $A_{1}\left(A_{2}\right.$, resp.) at the point $y$. By the hypothesis the angle between the directions of the strips $\mathcal{S}_{1}, \mathcal{S}_{2}$ is $\geq \ell / 10 \geq 100 R^{-1 / 2}$. Hence, $\operatorname{diam}\left(\mathcal{S}_{1} \cap \mathcal{S}_{2}\right) \leq 10 \ell^{-1} \leq R^{1 / 2} / 100$. Also note that $A_{i} \cap B\left(y, R^{1 / 2}\right) \subset \mathcal{S}_{i}$ for $i=1,2$. Since $\mathcal{C}$ is connected, it follows that $\mathcal{C} \subset \mathcal{S}_{1} \cap \mathcal{S}_{2}$. Thus $\operatorname{diam}(\mathcal{C}) \lesssim \ell^{-1}$

Proof of Theorem 3. It suffices to prove the theorem for $q \geq 2$ (see Remark 2 in the introduction). We give a proof for $q=2$ only. The proof for $q>2$ can be obtained by modifying the proof for $q=2$ as in the proof of Theorem 5 below. Without loss of generality let $C_{1}=1$. Note that it suffices to prove the theorem for $A_{R}(1,[-1 / 2,1 / 2])$ instead of $A_{R}(1)$.

Let $f$ be a function supported in $A_{R}(1,[-1 / 2,1 / 2])$ with $\|f\|_{2}=1$. We utilize the bilinear approach (see, e.g., [9], [12], [8]). Consider the set of dyadic intervals in $[-1 / 2,1 / 2]$. We say two dyadic intervals $I, J$ are related, $I \sim J$, if i) they have the same length, ii) they are not adjacent and iii) their parents are adjacent. Note that

$$
[-1 / 2,1 / 2] \times[-1 / 2,1 / 2]=\left[\bigcup_{1 \leq 2^{n} \leq R^{1 / 2}}\left[\bigcup_{|I|=|J|=2^{-n}, I \sim J}(I \times J)\right]\right] \bigcup \mathcal{D}
$$

Here $\mathcal{D}$ is a subset of the $C R^{-1 / 2}$-neighborhood of the diagonal $\{(x, x): x \in$ $[-1 / 2,1 / 2]\}$ which can be written as a union of finitely overlapping boxes $I \times I$ of side length $\approx R^{-1 / 2}$. Let $f_{I}:=f \chi_{A_{R}(1, I)}$. Using the decomposition (15), it is easy to see that

$$
\left(f^{\vee}\right)^{2}(\xi)=\sum_{n=0}^{\log \left(R^{1 / 2}\right)} \sum_{|I|=|J|=2^{-n}, I \sim J} f_{I}^{\vee}(\xi) f_{J}^{\vee}(\xi)+\text { Error },
$$

where

$$
\mid \text { Error }\left.\left|\lesssim \sum_{I \in I_{E}}\right| f_{I}^{\vee}(\xi)\right|^{2}
$$


Here $I_{E}$ is a set of finitely overlapping intervals of length $\approx R^{-1 / 2}$. By "finitely overlapping", we mean that $\left\|\sum_{I \in I_{E}} \chi_{I}\right\|_{\infty} \lesssim 1$. Using (16), we have

$$
\begin{aligned}
\left\|f^{\vee}\right\|_{L^{2}(\mu)}^{2} & \leq \sum_{n=0}^{\log \left(R^{1 / 2}\right)} \sum_{|I|=|J|=2^{-n}, I \sim J}\left\|f_{I}^{\vee} f_{J}^{\vee}\right\|_{L^{1}(\mu)}+\sum_{I \in I_{E}}\left\|f_{I}^{\vee}\right\|_{L^{2}(\mu)}^{2} \\
& =: S_{1}+S_{2} .
\end{aligned}
$$

Note that for each $I \in I_{E}$, the support of $f_{I}, A_{R}(1, I)$, is contained in a rectangle $D$ of dimensions $C \times C R^{1 / 2}$. Hence $f_{I}^{\vee}=f_{I}^{\vee} * \varphi_{D}^{\vee}$. Using this and Hölder's inequality, we have

$$
\left|f_{I}^{\vee}\right| \leq\left(\left|f_{I}^{\vee}\right|^{2} *\left|\varphi_{D}^{\vee}\right|\right)^{1 / 2}\left\|\varphi_{D}^{\vee}\right\|_{1}^{1 / 2} \lesssim\left(\left|f_{I}^{\vee}\right|^{2} *\left|\varphi_{D}^{\vee}\right|\right)^{1 / 2}
$$

Using this and Fubini's theorem, we obtain

$$
\left\|f_{I}^{\vee}\right\|_{L^{2}(\mu)}^{2} \leq \int\left|f_{I}^{\vee}(x)\right|^{2}\left(\mu *\left|\varphi_{D}^{\vee}\right|\right)(x) d x \lesssim\left\|f_{I}\right\|_{2}^{2} R^{1-\alpha / 2}
$$

In the last inequality, we used Lemma 3.1(I) and Parseval's theorem. Since the intervals in $I_{E}$ are finitely overlapping, (18) implies that

$$
S_{2} \lesssim R^{1-\alpha / 2}
$$

To complete the proof of the theorem, we should obtain the same bound for $S_{1}$. Since there are $\lesssim \log (R)$ values of $n$ and orthogonality (see, e.g., [9], [12]), it suffices to prove that for each $n$ and for each pair $I \sim J,|I|=|J|=2^{-n}$,

$$
\left\|f_{I}^{\vee} f_{J}^{\vee}\right\|_{L^{1}(\mu)} \lesssim R^{1-\alpha / 2}\left\|f_{I}\right\|_{2}\left\|f_{J}\right\|_{2}
$$

where the implicit constant is independent of $I, J$ and $R$. To prove (19), first note that the union of the supports of $f_{I}$ and $f_{J}$ are contained in a rectangle of dimensions $C R 2^{-n} \times C R 2^{-2 n}$. Hence, $f_{I} * f_{J}$ is supported in a rectangle $D$ of dimensions $2 C R 2^{-n} \times 2 C R 2^{-2 n}$, the longer axis being in the direction $e$, say. Using $f_{I} * f_{J}=\left(f_{I} * f_{J}\right) \varphi_{D}$ like above, we obtain

$$
\left\|f_{I}^{\vee} f_{J}^{\vee}\right\|_{L^{1}(\mu)} \leq \int\left|f_{I}^{\vee}(x) f_{J}^{\vee}(x)\right|\left(\mu *\left|\varphi_{D}^{\vee}\right|\right)(x) d x .
$$

Consider a tiling of $\mathbf{R}^{2}$ with rectangles $P$ of dimensions $100 \times 1002^{-n}$, the short axis being in the direction of $e$. Note that each $P$ is contained in a rectangle $x_{P}+C R 2^{-2 n} D_{d u a l}$ for some $x_{P} \in \mathbf{R}^{2}$. Using the properties of the function $\phi$, we obtain

$$
1 \lesssim \sum_{P} \phi_{P}^{3} \lesssim \sum_{P} \phi_{P}^{2} \lesssim 1
$$


Let $f_{I, P}:=\widehat{f_{I}^{\vee} \phi_{P}}$. Using $(21)$ in $(20)$ and then Cauchy-Schwarz inequality, we get

$$
\begin{aligned}
(20) & \lesssim \sum_{P} \int\left|f_{I, P}^{\vee}(x) f_{J, P}^{\vee}(x)\right|\left(\mu *\left|\varphi_{D}^{\vee}\right|\right)(x) \phi_{P}(x) \mathrm{d} x \\
& \lesssim \sum_{P}\left[\int\left|f_{I, P}^{\vee}(x) f_{J, P}^{\vee}(x)\right|^{2} \mathrm{~d} x\right]^{1 / 2}\left[\int\left[\left(\mu *\left|\varphi_{D}^{\vee}\right|\right)(x) \phi_{P}(x)\right]^{2} \mathrm{~d} x\right]^{1 / 2}
\end{aligned}
$$

To estimate the first integral in (22), we use a well-known $L^{4}$ orthogonality argument, see, e.g., [8]. Let $A_{I, P}$ be the support of $f_{I, P}$. By Parseval's theorem and Cauchy-Schwarz, Young's inequalities

$$
\begin{aligned}
\int\left|f_{I, P}^{\vee}(x) f_{J, P}^{\vee}(x)\right|^{2} \mathrm{~d} x & =\int\left|f_{I, P} * f_{J, P}(\xi)\right|^{2} \mathrm{~d} \xi \\
& \lesssim\left\|\left|\left(\xi+A_{I, P}\right) \cap A_{J, P}\right|\right\|_{L^{\infty}(\xi)} \int\left(\left|f_{I, P}\right|^{2} *\left|f_{J, P}\right|^{2}\right)(\xi) \mathrm{d} \xi \\
& \lesssim\left\|\left|\left(\xi+A_{I, P}\right) \cap A_{J, P}\right|\right\|_{L^{\infty}(\xi)}\left\|f_{I, P}\right\|_{2}^{2}\left\|f_{J, P}\right\|_{2}^{2} .
\end{aligned}
$$

Note that $f_{I, P}=f_{I} * \widehat{\phi_{P}}$. Hence $A_{I, P}$ is contained in $\operatorname{supp}\left(f_{I}\right)+\operatorname{supp}\left(\widehat{\phi_{P}}\right) \subset$ $\operatorname{supp}\left(f_{I}\right)+P_{\text {dual }}$, where $P_{\text {Dual }}$ is the dual of $P$ centered at the origin. At this point the crucial observation is the following:

$$
\operatorname{supp}\left(f_{I}\right)+P_{\text {dual }} \subset A_{R}\left(10, \frac{11}{10} I\right) .
$$

Thus, Lemma 3.2 implies that $\left\|\left|\left(\xi+A_{I, P}\right) \cap A_{J, P}\right|\right\|_{L^{\infty}(\xi)} \lesssim|I|^{-1}=2^{n}$. Using this in (23), we see that

$$
\int\left|f_{I, P}^{\vee}(x) f_{J, P}^{\vee}(x)\right|^{2} \mathrm{~d} x \lesssim 2^{n}\left\|f_{I, P}\right\|_{2}^{2}\left\|f_{J, P}\right\|_{2}^{2}
$$

Now, we obtain a bound for the second integral in (22). This is just a simple application of Lemma 3.1. First note that by Lemma 3.1(I), we have

$$
\left\|\mu * \mid \varphi_{D}^{\vee}\right\|_{\infty} \lesssim R^{2-\alpha} 2^{n \alpha-2 n} .
$$

Second, using (13) for $\phi_{P}$ and Lemma 3.1(III) (remember that $P$ is contained in $x_{P}+C R 2^{-2 n} D_{d u a l}$ for some $x_{P} \in \mathbf{R}^{2}$ ), we have

$$
\begin{aligned}
\int\left(\mu *\left|\varphi_{D}^{\vee}\right|\right)(x) \phi_{P}(x) \mathrm{d} x & \leq \sum_{j=1}^{\infty} 2^{-M j} \int\left(\mu *\left|\varphi_{D}^{\vee}\right|\right)(x) \chi_{2^{j} P}(x) \mathrm{d} x \\
& \lesssim \sum_{j=1}^{\infty} 2^{-M j} 2^{n-n \alpha} 2^{j \alpha} \lesssim 2^{n-n \alpha} .
\end{aligned}
$$

Using (25) and (26), we have

$$
\begin{aligned}
\int\left[\left(\mu *\left|\varphi_{D}^{\vee}\right|\right)(x) \phi_{P}(x)\right]^{2} \mathrm{~d} x & \lesssim\left\|\mu *\left|\varphi_{D}^{\vee}\right|\right\|_{\infty} \int\left(\mu *\left|\varphi_{D}^{\vee}\right|\right)(x) \phi_{P}(x) \mathrm{d} x \\
& \lesssim R^{2-\alpha} 2^{-n} .
\end{aligned}
$$


Substituting (24) and (27) into (22) yields (19):

$$
\begin{aligned}
\left\|f_{I}^{\vee} f_{J}^{\vee}\right\|_{L^{1}(\mu)} & \lesssim R^{1-\alpha / 2} \sum_{P}\left\|f_{I, P}\right\|_{2}\left\|f_{J, P}\right\|_{2} \\
& \lesssim R^{1-\alpha / 2}\left[\sum_{P}\left\|f_{I, P}\right\|_{2}^{2}\right]^{1 / 2}\left[\sum_{P}\left\|f_{J, P}\right\|_{2}^{2}\right]^{1 / 2} \\
& \lesssim R^{1-\alpha / 2}\left\|f_{I}\right\|_{2}\left\|f_{J}\right\|_{2} .
\end{aligned}
$$

In the last inequality, we used Parseval's theorem and (21).

\section{Proof of Theorem 5}

We prove the theorem for $\alpha \in[1,2]$ (see Remark 6 in the introduction). We have the following analog of Lemma 3.1.

Lemma 4.1. Let $\alpha \in[1,2]$. Let $\mu$ be a non-negative measure in $\mathbf{R}^{3}$ which satisfies (10) with $C_{1}=1$. Let $D$ be a rectangle of dimensions $R_{1} \times R_{2} \times R_{3}$ such that $R_{1} \leq R_{2} \leq R_{3} \lesssim R$. Let $D_{\text {dual }}$ be the dual of $D$ centered at the origin. Then the function $\mu_{D}:=\left|\varphi_{D}^{\vee}\right| * \mu$ satisfies

I) $\left\|\mu_{D}\right\|_{\infty} \lesssim R_{2}^{2-\alpha} R_{3}$,

II) $\left\|\mu_{D}\right\|_{1} \lesssim 1$

III) $\mu_{D}\left(x+K D_{\text {dual }}\right):=\int_{K D_{\text {dual }}} \mu_{D}(x+y) d y \lesssim K^{\alpha} R_{2}^{1-\alpha} R_{1}^{-1}, \forall K \gtrsim 1$ and $x \in \mathbf{R}^{3}$.

We omit the proof since it is similar to the proof of Lemma 3.1.

Proof of Theorem 5. The proof is similar to the proof of Theorem 3. We can assume that $q \geq 2$ and $C_{1}=1$. Let $\Gamma_{R}(C, J):=\left\{(\rho \cos (\theta), \rho \sin (\theta), t) \in \Gamma_{R}(C)\right.$ : $\theta \in J\}$. It suffices to prove the theorem with $\Gamma_{R}(1,[-1 / 2,1 / 2])$ instead of $\Gamma_{R}(1)$. Let $f$ be a function supported in $\Gamma_{R}(1,[-1 / 2,1 / 2])$ with $\|f\|_{2}=1$. Given interval $I$, let $f_{I}:=f \chi_{\Gamma_{R}(1, I)}$. Using the decomposition (15) as in the proof of Theorem 3, we obtain

$$
\left|f^{\vee}(\xi)\right|^{2} \leq \sum_{n} \sum_{|I|=|J|=2^{-n}, I \sim J}\left|f_{I}^{\vee}(\xi) f_{J}^{\vee}(\xi)\right|+\sum_{I \in I_{E}}\left|f_{I}^{\vee}\right|^{2},
$$

where, $I_{E}$ is a set of finitely overlapping intervals of length $\approx R^{-1 / 2}$. Using $(28)$, we have for any $q \geq 2$

$$
\begin{aligned}
\left\|f^{\vee}\right\|_{L^{q}(\mu)}^{2} & \leq \sum_{n=1}^{\log \left(R^{1 / 2}\right)} \sum_{|I|=|J|=2^{-n}, I \sim J}\left\|f_{I}^{\vee} f_{J}^{\vee}\right\|_{L^{q / 2}(\mu)}+\sum_{I \in I_{E}}\left\|f_{I}^{\vee}\right\|_{L^{q}(\mu)}^{2} \\
& =: S_{1}+S_{2} .
\end{aligned}
$$

Note that for each $I \in I_{E}$, the support of $f_{I}, \Gamma_{R}(1, I)$, is contained in a rectangle $D$ of dimensions $C \times C R^{1 / 2} \times C R$. Hence $f_{I}^{\vee}=f_{I}^{\vee} * \varphi_{D}^{\vee}$. Using this and Hölder's inequality, we have

$$
\left|f_{I}^{\vee}\right| \leq\left(\left|f_{I}^{\vee}\right|^{q} *\left|\varphi_{D}^{\vee}\right|\right)^{1 / q}\left\|\varphi_{D}^{\vee}\right\|_{1}^{1-1 / q} \lesssim\left(\left|f_{I}^{\vee}\right|^{q} *\left|\varphi_{D}^{\vee}\right|\right)^{1 / q}
$$


Using this, Fubini's theorem and Hausdorff-Young inequality, we obtain

$$
\begin{aligned}
\left\|f_{I}^{\vee}\right\|_{L^{q}(\mu)}^{q} & \leq \int\left|f_{I}^{\vee}(x)\right|^{q}\left(\mu *\left|\varphi_{D}^{\vee}\right|\right)(x) \mathrm{d} x \lesssim\left\|f_{I}\right\|_{q^{\prime}}^{q}\left\|\mu *\left|\varphi_{D}^{\vee}\right|\right\|_{\infty} \\
& \lesssim\left\|f_{I}\right\|_{2}^{q} R^{\frac{3}{2}\left(\frac{q}{2}-1\right)} R^{2-\alpha / 2} .
\end{aligned}
$$

In the last inequality, we used Lemma 4.1(I) and Hölder's inequality. Since the intervals in $I_{E}$ are finitely overlapping (18) implies that

$$
S_{2} \lesssim R^{\frac{3}{2}+\frac{1-\alpha}{q}}
$$

This bound takes care of $S_{2}$ for any $q \geq 2$. In what follows, we obtain bounds for $S_{1}$ for $q=2$ and $q \geq 4$, the remaining case follows from interpolation.

Case 1) $q=2$.

As in the proof of Theorem 3, it suffices to prove that for each $n$ and for each pair $I \sim J,|I|=|J|=2^{-n}$,

$$
\left\|f_{I}^{\vee} f_{J}^{\vee}\right\|_{L^{1}(\mu)} \lesssim R^{2-\alpha / 2}\left\|f_{I}\right\|_{2}\left\|f_{J}\right\|_{2}
$$

where the implicit constant is independent of $I, J$ and $R$. Note that the union of the supports of $f_{I}$ and $f_{J}$ are contained in a rectangle of dimensions $C R \times$ $C R 2^{-n} \times C R 2^{-2 n}$. Hence $f_{I} * f_{J}$ is supported in a rectangle $D$ of dimensions $2 C R \times 2 C R 2^{-n} \times 2 C R 2^{-2 n}$, the longest axis being in the direction $e$ and the second longest axis being in the direction of $f$, say. Note that $e$ is a light direction and $f$ is tangent to the light cone at $e$. Like above, we have

$$
\left\|f_{I}^{\vee} f_{J}^{\vee}\right\|_{L^{1}(\mu)} \leq \int\left|f_{I}^{\vee}(x) f_{J}^{\vee}(x)\right|\left(\mu *\left|\varphi_{D}^{\vee}\right|\right)(x) d x .
$$

Let $T_{e}$ be the Lorentz transformation (see, e.g., [11], [12]) satisfying

$$
T_{e}(e)=e, \quad T_{e}(f)=2^{n} f, \quad T_{e}(e \times f)=2^{2 n} e \times f .
$$

Let $F_{I}(\xi)=f_{I}\left(T_{e}^{-1}(\xi)\right)$ and $F_{J}(\xi)=f_{J}\left(T_{e}^{-1}(\xi)\right)$. Note that $F_{I}$ is supported in $\Gamma_{R}\left(2^{2 n}, I^{\prime}\right)$ and $F_{J}$ is supported in $\Gamma_{R}\left(2^{2 n}, J^{\prime}\right)$, where $I^{\prime}$ and $J^{\prime}$ are intervals of length $\approx 1$ and the distance between them is $\approx 1$. Also note that

$$
\begin{aligned}
f_{I}^{\vee}(x) & =F_{I}^{\vee}\left(T_{e}^{-1}(x)\right) 2^{-3 n}, \\
\left\|f_{I}\right\|_{2} & =\left\|F_{I}\right\|_{2} 2^{-3 n / 2} .
\end{aligned}
$$

Substituting (33) in (32) and then changing the variable, we get

$$
\left\|f_{I}^{\vee} f_{J}^{\vee}\right\|_{L^{1}(\mu)} \lesssim 2^{-3 n} \int\left|F_{I}^{\vee}(u) F_{J}^{\vee}(u)\right|\left(\mu *\left|\varphi_{D}^{\vee}\right|\right)\left(T_{e}(u)\right) \mathrm{d} u .
$$

Consider a tiling of $\mathbf{R}^{3}$ with boxes $P$ of side length $1002^{-2 n}$. Note that (21) is valid for $\phi_{P}$. Let $F_{I, P}:=\widehat{f_{I}^{\vee} \phi_{P}}$. Using $(21)$ in (34) and then Hölder's inequality, 
we obtain

$$
\begin{aligned}
\left\|f_{I}^{\vee} f_{J}^{\vee}\right\|_{L^{1}(\mu)} & \lesssim 2^{-3 n} \sum_{P} \int\left|F_{I, P}^{\vee}(u) F_{J, P}^{\vee}(u)\right|\left(\mu *\left|\varphi_{D}^{\vee}\right|\right)\left(T_{e}(u)\right) \phi_{P}(u) \mathrm{d} u \\
& \lesssim 2^{-3 n} \sum_{P}\left[\int\left|F_{I, P}^{\vee}(u) F_{J, P}^{\vee}(u)\right|^{2} \mathrm{~d} u\right]^{1 / 2} \cdot \\
& {\left[\int\left[\left(\mu *\left|\varphi_{D}^{\vee}\right|\right)\left(T_{e}(u)\right) \phi_{P}(u)\right]^{2} \mathrm{~d} u\right]^{1 / 2} }
\end{aligned}
$$

We estimate the first integral in (35) as in the proof of Theorem 3. Let $A_{I, P}$ be the support of $F_{I, P}$. By Parseval's theorem and Cauchy-Schwarz, Young's inequalities

$$
\begin{aligned}
\int\left|F_{I, P}^{\vee}(u) F_{J, P}^{\vee}(u)\right|^{2} \mathrm{~d} u & =\int\left|F_{I, P} * F_{J, P}(\xi)\right|^{2} \mathrm{~d} \xi \\
& \lesssim\left\|\left|\left(\xi+A_{I, P}\right) \cap A_{J, P}\right|\right\|_{L^{\infty}(\xi)} \int\left(\left|F_{I, P}\right|^{2} *\left|F_{J, P}\right|^{2}\right)(\xi) \mathrm{d} \xi \\
& \lesssim\left\|\left|\left(\xi+A_{I, P}\right) \cap A_{J, P}\right|\right\|_{L^{\infty}(\xi)}\left\|F_{I, P}\right\|_{2}^{2}\left\|F_{J, P}\right\|_{2}^{2} .
\end{aligned}
$$

Like before $A_{I, P}$ is contained in $\operatorname{supp}\left(F_{I}\right)+\operatorname{supp}\left(\widehat{\phi_{P}}\right) \subset \operatorname{supp}\left(F_{I}\right)+P_{\text {dual }}$, where $P_{d u a l}$ is a cube of side length $2^{2 n} / 100$ centered at the origin. Thus $A(I, P)$ is contained in $\Gamma_{R}\left(C 2^{2 n}, \frac{11}{10} I^{\prime}\right)$. The transversality of the cone (see, e.g., [11], [12]) implies that $\left\|\left|\left(\xi+A_{I, P}\right) \cap A_{J, P}\right|\right\|_{L^{\infty}(\xi)} \lesssim R 2^{4 n}$. Using this in (36), we see that

$$
\int\left|F_{I, P}^{\vee}(u) F_{J, P}^{\vee}(u)\right|^{2} \mathrm{~d} u \lesssim R 2^{4 n}\left\|F_{I, P}\right\|_{2}^{2}\left\|F_{J, P}\right\|_{2}^{2} .
$$

Now, we obtain a bound for the second integral in (35).

$$
\begin{aligned}
\int\left[\left(\mu *\left|\varphi_{D}^{\vee}\right|\right)\left(T_{e}(u)\right) \phi_{P}(u)\right]^{2} \mathrm{~d} u & \lesssim 2^{-3 n}\left\|\mu *\left|\varphi_{D}^{\vee}\right|\right\|_{\infty} \int\left(\mu *\left|\varphi_{D}^{\vee}\right|\right)(u) \phi_{P}\left(T_{e}^{-1}(u)\right) \mathrm{d} u \\
& =2^{-3 n}\left\|\mu *\left|\varphi_{D}^{\vee}\right|\right\|_{\infty} \int\left(\mu *\left|\varphi_{D}^{\vee}\right|\right)(u) \phi_{T_{e}(P)}(u) \mathrm{d} u
\end{aligned}
$$

By Lemma 4.1(I), we have

$$
\left\|\mu *\left|\varphi_{D}^{\vee}\right|\right\|_{\infty} \lesssim R^{3-\alpha} 2^{n \alpha-2 n} .
$$

Note that $T_{e}(P)$ has dimensions $C 2^{-2 n} \times C 2^{-n} \times C$ and it is a multiple of a dual of $D$. Thus we can apply Lemma 4.1(III) to obtain

$$
\int\left(\mu *\left|\varphi_{D}^{\vee}\right|\right)(u) \phi_{T_{e}(P)}(u) \mathrm{d} u \lesssim 2^{n-n \alpha}
$$

Using (39) and (40) in (38), we have

$$
\int\left[\left(\mu *\left|\varphi_{D}^{\vee}\right|\right)\left(T_{e}(u)\right) \phi_{P}(u)\right]^{2} \mathrm{~d} u \lesssim R^{3-\alpha} 2^{-4 n} .
$$


Substituting (37) and (41) into (35) and then using (33), we obtain (31):

$$
\begin{aligned}
\left\|f_{I}^{\vee} f_{J}^{\vee}\right\|_{L^{1}(\mu)} & \lesssim R^{2-\alpha / 2} 2^{-3 n} \sum_{P}\left\|F_{I, P}\right\|_{2}\left\|F_{J, P}\right\|_{2} \\
& \lesssim R^{2-\alpha / 2} 2^{-3 n}\left[\sum_{P}\left\|F_{I, P}\right\|_{2}^{2}\right]^{1 / 2}\left[\sum_{P}\left\|F_{J, P}\right\|_{2}^{2}\right]^{1 / 2} \\
& \lesssim R^{2-\alpha / 2} 2^{-3 n}\left\|F_{I}\right\|_{2}\left\|F_{J}\right\|_{2}=R^{2-\alpha / 2}\left\|f_{I}\right\|_{2}\left\|f_{J}\right\|_{2} .
\end{aligned}
$$

Case 2) $q \geq 4$.

Similarly, we have

$$
\left\|f_{I}^{\vee} f_{J}^{\vee}\right\|_{L^{q / 2}(\mu)}^{q / 2} \leq \int\left|f_{I}^{\vee}(x) f_{J}^{\vee}(x)\right|^{q / 2}\left(\mu *\left|\varphi_{D}^{\vee}\right|\right)(x) d x
$$

where $D$ is a rectangle of dimensions $C R \times C R 2^{-n} \times C R 2^{-2 n}$. Using (39), we have

$$
\begin{aligned}
\left\|f_{I}^{\vee} f_{J}^{\vee}\right\|_{L^{q / 2}(\mu)}^{q / 2} & \lesssim R^{3-\alpha} 2^{n \alpha-2 n} \int\left|f_{I}^{\vee}(x) f_{J}^{\vee}(x)\right|^{q / 2} \mathrm{~d} x \\
& \lesssim R^{3-\alpha} 2^{n \alpha-2 n}\left\|f_{I}^{\vee} f_{J}^{\vee}\right\|_{\infty}^{\frac{q}{2}-2} \int\left|f_{I}^{\vee}(x) f_{J}^{\vee}(x)\right|^{2} \mathrm{~d} x
\end{aligned}
$$

Using the Lorentz transformation $T_{e}$ as in case 1 , we have

$$
\int\left|f_{I}^{\vee}(x) f_{J}^{\vee}(x)\right|^{2} \mathrm{~d} x \lesssim R 2^{n}\left\|f_{I}\right\|_{2}^{2}\left\|f_{J}\right\|_{2}^{2}
$$

We also have

$$
\left\|f_{I}^{\vee} f_{J}^{\vee}\right\|_{\infty} \leq\left\|f_{I} * f_{J}\right\|_{1} \leq\left\|f_{I}\right\|_{1}\left\|f_{J}\right\|_{1} \lesssim R^{2} 2^{-n}\left\|f_{I}\right\|_{2}\left\|f_{J}\right\|_{2}
$$

Substituting (44) and (45) into (43), we have

$$
\left\|f_{I}^{\vee} f_{J}^{\vee}\right\|_{L^{q / 2}(\mu)} \lesssim R^{2-\frac{2 \alpha}{q}} 2^{n\left(\frac{2}{q}(\alpha+1)-1\right)}\left\|f_{I}\right\|_{2}\left\|f_{J}\right\|_{2}
$$

Note that if $\alpha \geq \frac{q}{2}-1$, then $\frac{2}{q}(\alpha+1)-1 \geq 0$ and hence $R^{2-\frac{2 \alpha}{q}} 2^{n\left(\frac{2}{q}(\alpha+1)-1\right)} \lesssim$ $R^{\frac{3}{2}-\frac{\alpha-1}{q}}$. Otherwise, $R^{2-\frac{2 \alpha}{q}} 2^{n\left(\frac{2}{q}(\alpha+1)-1\right)} \lesssim R^{2-\frac{2 \alpha}{q}}$. Using these two inequalities in (46), we have

$$
\left\|f_{I}^{\vee} f_{J}^{\vee}\right\|_{L^{q / 2}(\mu)} \lesssim R^{\max \left(2-\frac{2 \alpha}{q}, \frac{3}{2}-\frac{\alpha-1}{q}\right)}\left\|f_{I}\right\|_{2}\left\|f_{J}\right\|_{2} .
$$

Substituting (47) into (29) yields the required bound.

\section{Appendix}

In this appendix, we prove (8) and Theorem 1.

Let $S=\left\{x \in \mathbf{R}^{3}: x_{3} \in[-2 R, 2 R],\left|x_{1}-x_{3}\right|<1,\left|x_{2}\right|<R^{1 / 2}\right\}$. Let $\psi_{R}$ be a function satisfying i) $\left\|\psi_{R}\right\|_{1} \approx 1$, ii) it is supported in a rectangle of dimensions $C \times C R^{-1 / 2} \times C R^{-1}$, iii) $\widehat{\psi_{R}} \in[1 / 2,1]$ in $S$, iv) $\widehat{\psi_{R}}$ has a Schwartz decay away from $S$. 
Let $\mathrm{d} \mu(x)=\psi_{R}(x) \mathrm{d} x$. Note that

$$
\int_{\Gamma}|\widehat{\mu}(R u)|^{2} \mathrm{~d} \nu_{\Gamma}(u) \gtrsim R^{-1 / 2}
$$

Also note that

$$
I_{\alpha}(\mu) \approx \int \frac{\widehat{\psi_{R}}(\xi)^{2}}{|\xi|^{3-\alpha}} \mathrm{d} \xi \lesssim \begin{cases}1 & , \alpha \in(0,1] \\ R^{\frac{\alpha-1}{2}} & , \alpha \in[1,2] \\ R^{\alpha-3 / 2} & , \alpha \in[2,3] .\end{cases}
$$

It is easy to obtain the last inequality if one replaces $\widehat{\psi_{R}}$ with the characteristic function of $S$. The inequality follows from the Schwartz decay of $\widehat{\psi_{R}}$ away from $S$. Note that (48) and (49) imply (8) for $\alpha \geq 1 / 2$. To prove that $\beta(\alpha) \leq \alpha$, one may use the functions $f_{\eta}(x)=|x|^{-\eta}$. Fix $\alpha_{1} \in(\alpha, 3)$ and let $\mathrm{d} \mu(x)=$ $f_{3-\alpha_{1} / 2}(x) \psi_{1}(x) \mathrm{d} x$, where $\psi_{1}=\psi_{R=1}$. Then

$$
\widehat{\mu}(\xi)=c f_{\alpha_{1} / 2} * \widehat{\psi_{1}}(\xi) \approx(1+|\xi|)^{-\alpha_{1} / 2} .
$$

Thus $I_{\alpha}(\mu) \approx 1$ and $\int_{\Gamma}|\widehat{\mu}(R u)|^{2} \mathrm{~d} \nu(u) \approx R^{-\alpha_{1}}$, which imply that $\beta(\alpha) \leq \alpha$.

Proof of Theorem 1. [6] The following calculation yields the first bound. It suffices to consider the case $\alpha \leq a$.

$$
\begin{aligned}
\int|\widehat{\mu}(R u)|^{2} \mathrm{~d} \nu(u) & =\int \widehat{\nu}(R(x-y)) \mathrm{d} \mu(x) \mathrm{d} \mu(y) \lesssim \int \frac{\mathrm{d} \mu(x) \mathrm{d} \mu(y)}{(1+R|x-y|)^{a}} \\
& \leq \int \frac{\mathrm{d} \mu(x) \mathrm{d} \mu(y)}{(R|x-y|)^{\alpha}}=R^{-\alpha} I_{\alpha}(\mu) .
\end{aligned}
$$

Second bound follows from the uncertainty principle. Using $\mathrm{d} \mu(x)=\varphi(x) \mathrm{d} \mu(x)$ (see Section 2 for the definition of $\varphi$ ), we get

$$
\widehat{\mu}(u)=\widehat{\mu} * \widehat{\varphi}(u) .
$$

Using Cauchy-Schwarz inequality, we obtain

$$
\begin{aligned}
\int|\widehat{\mu}(R u)|^{2} \mathrm{~d} \nu(u) & =\int\left|\int \widehat{\mu}(\xi) \widehat{\varphi}(R u-\xi) \mathrm{d} \xi\right|^{2} \mathrm{~d} \nu(u) \\
& \leq\|\widehat{\varphi}\|_{L^{1}} \int|\widehat{\mu}(\xi)|^{2}|\widehat{\varphi}(R u-\xi)| \mathrm{d} \xi \mathrm{d} \nu(u) \\
& \lesssim I_{\alpha}(\mu) \sup _{\xi}\left(|\xi|^{d-\alpha} \int|\widehat{\varphi}(R u-\xi)| \mathrm{d} \nu(u)\right) .
\end{aligned}
$$

Note that the Schwartz decay, $|\widehat{\varphi}(x)| \leq C_{M}(1+|x|)^{-M}$, and the density assumption on $\nu$ imply that

$$
\int|\widehat{\varphi}(R u-\xi)| \mathrm{d} \nu(u) \lesssim\left\{\begin{array}{ll}
R^{-b} & ,|\xi| \lesssim R \\
|\xi|^{-M} & ,|\xi|>>R
\end{array}\right\} \lesssim R^{d-\alpha-b}|\xi|^{\alpha-d}
$$

if $M$ has been chosen large enough. Substituting (51) in (50) yields the second bound. 


\section{Acknowledgments}

This work was partially supported by NSF grant DMS-0303413. The author wishes to thank Michael Christ for many useful conversations.

\section{References}

[1] J. Bourgain, Hausdorff dimension and distance sets, Israel J. Math. 87 (1994), 193-201.

[2] K.J. Falconer, The geometry of fractal sets, Cambridge Tracts in Math. 85, Cambridge University Press, 1985.

[3] _ On the Hausdorff dimension of distance sets, Mathematika 32 (1985), 206-212.

[4] P. Mattila, Spherical averages of Fourier transforms of measures with finite energy: dimension of intersections and distance sets, Mathematika 34 (1987), 207-228.

[5] P. Sjölin, Estimates of spherical averages of Fourier transforms and dimensions of sets, Mathematika 40 (1993), 322-330.

[6] _ Estimates of averages of Fourier transforms of measures with finite energy Ann. Acad. Sci. Fenn. Math. 22 (1997), 227-236.

[7] T. Tao, A sharp bilinear restriction estimate for paraboloids, GAFA 13 (2003), 1359-1384.

[8] - Some recent progress on the restriction conjecture, preprint.

[9] _ A. Vargas, L. Vega, A bilinear approach to the restriction and Kakeya conjectures, J. Amer. Math. Soc. 11 (1998), 967-5000.

[10] T. Wolff, Decay of circular means of Fourier transforms of measures, IMRN, 1999, 547567.

[11] Local smoothing type estimates on $L^{p}$ for large p, GAFA 10 (2000), 1237-1288.

[12] _ A sharp bilinear cone restriction estimate, Ann. of Math. (2) 153 (2001), 661-698.

Department of Mathematics, University of California, Berkeley, CA 94720-3840

E-mail address: burak@math.berkeley.edu 